\title{
Development of an intraruminal device for data sampling and transmission
}

\author{
A. K. Sievers ${ }^{1}$, N. B. Kristensen ${ }^{2}$, H.-J. Laue ${ }^{3}$ and S. Wolffram ${ }^{1,4}$ \\ ${ }^{1}$ Institute of Animal Nutrition, Physiology and Metabolism, \\ Christian-Albrechts-University, 24098 Kiel, Germany \\ ${ }^{2}$ Department of Animal Nutrition and Physiology, Danish Institute of Agricultural Sciences \\ P.O. Box 50, DK-8830 Tjele, Denmark \\ ${ }^{3}$ Department of Cultivation, University of Applied Sciences \\ Kiel 24783, Osterrönfeld, Germany
}

\begin{abstract}
The number of animals within European dairy herds is increasing and the time for surveillance of each cow decreases. The aim of the present study was to develop an intraruminal device for monitoring changes in conductivity, pressure, and temperature in individual cows. The precision and accuracy of the device was evaluated by in vitro and in vivo experiments. Whereas conductivity was increased by volatile fatty acid anions concentration-dependently, it was reduced by an increase in osmolarity. For changes in temperature, conductivity and pressure, high correlations were observed between values obtained by the intraruminal device and those registered by external reference methods. The intraruminal device might be of benefit to future feed and management systems for dairy cows.
\end{abstract}

KEY WORDS: intraruminal device, dairy cattle, management, conductivity, pressure, temperature

\section{INTRODUCTION}

The situation on dairy farms has noticeably changed over the years. Mainly due to economical reasons the number of animals within a dairy herd is constantly increasing. The increased number of animals per herd is usually accompanied by a decrease of monitoring time per animal. As a consequence health disturbances and oestrus of individual cows might not be perceived in time. This might reduce profitability due to decreased milk production and additional costs for the veterinarian and artificial insemination (Lehrer et al., 1992). An optimized management system with little or no need for an additional expenditure of time can improve the health status of the herd and the economic situation of the farm.

\footnotetext{
${ }^{1}$ Corresponding author: e-mail: wolffram@aninut.uni-kiel.de
} 
Using an intraruminal system would have clear advantages over other automatic or semi-automatic management systems like ear tag transponders or pedometers for the following reasons: an intraruminal device is independent of external disturbing factors, it can not be manipulated from the outside and is less likely to be lost. Furthermore such a device allows for a continuous monitoring of variables related to ruminal fermentation in individual cows. The disadvantage of the system is that an intraruminal device cannot be easily replaced. The aim of the present study was to develop an intraruminal device for measuring fermentationrelated parameters like conductivity, pressure changes and temperature, to store data temporarily and to transmit data periodically to a receiver unit.

\section{MATERIAL AND METHODS}

Together with a commercial company (Kneisner und Doering Elektronik $\mathrm{GmbH}$, Braunschweig, Germany) we have developed a prototype of a plastic bolus (weight $245 \mathrm{~g}$, specific weight $1.7 \mathrm{~g} / \mathrm{cm}^{3}$ ) with the following dimensions: $16 \mathrm{~cm}$ long and $3.4 \mathrm{~cm}$ outer diameter. It can be tightly sealed with a plastic cap. Within a small cavity at the bottom of the bolus a barometer (300 to $1300 \mathrm{hPa}$ ) is placed covered by a rubber membrane. The space between the membrane and the barometer is filled with a non-toxic fluid metal (Galinstan, Geratherm Medical $\mathrm{AG}$, Geschwenda, Germany) for pressure transmission. Inside the device there is an electrical conductivity meter $(0$ to $30 \mathrm{mS} / \mathrm{cm})$, a resistance thermometer $(-10$ to $60^{\circ} \mathrm{C}$; Altimetermodul MS5534/ Intersema, Switzerland), one printed board, a rechargeable battery as well as a chip for data storage and energy management. The conductivity meter has two fixed steel electrodes attached to the outside of the device.

For programming, the device is connected to a computer by a serial interface. For each instrument the number of measuring cycles, number of recordings within a measuring cycle and the duration of a measuring cycle can be individually recorded. The data are permanently stored and can be periodically transmitted to a computer for further evaluation.

In a first series of in vitro and in vivo experiments the principal functioning of the bolus was checked and the data obtained by the bolus were compared to those obtained by external reference devices. The thermometer as well as the barometer was examined with respect to sensitivity and velocity. The functioning of the conductivity meter was investigated with regard to the influence of individual volatile fatty acids (VFA), $\mathrm{pH}$, and osmolarity. The influence of VFA on conductivity recorded by the intraruminal device was tested by adding increasing concentrations of VFA as well as a mixture thereof to an artificial rumen fluid. Changes in osmolarity were provoked by the addition of various concentrations of D-mannitol. 
The barometer of the intraruminal device was checked by connecting a vertical silicon tube to the device. Accurately defined pressure changes were induced by changing the water filling of the tube $\left(1 \mathrm{~cm} \mathrm{H}_{2} \mathrm{O}=1 \mathrm{hPa}\right)$.

In addition, the intraruminal device has been tested in vivo in four ruminally cannulated lactating cows.

\section{RESULTS AND DISCUSSION}

Water temperatures could be recorded in vitro with an accuracy of $0.1^{\circ} \mathrm{C}$ without any recordable delay. Under in vivo conditions, a highly significant correlation was found between intraruminal temperature recordings by the device as compared with the rectal temperature $\left(n=36\right.$; Pearson $\left.r=0.92, R^{2}=0.84\right)$.

Acetic, butyric or propionic acid as well as a mixture of these VFA (molar ratio 6:3:1) increased the conductivity of the medium $(0 \mathrm{mmol} / \mathrm{L}: 12.76 \mathrm{mS} ; 50 \mathrm{mmol} / \mathrm{L}: 13.18$ $\mathrm{mS} ; 100 \mathrm{mmol} / \mathrm{L}: 13.94 \mathrm{mS} ; 150 \mathrm{mmol} / \mathrm{L}: 14.76 \mathrm{mS})$. No differences with respect to conductivity changes were obtained between individual VFA or the VFA mixture. The addition of VFA decreased $\mathrm{pH}(0 \mathrm{mmol} / \mathrm{L} \mathrm{pH} 6.88 ; 50 \mathrm{mmol} / \mathrm{L}: \mathrm{pH}$ 5.77; 100 $\mathrm{mmol} / \mathrm{L}: \mathrm{pH} 4.88 ; 150 \mathrm{mmol} / \mathrm{L}: \mathrm{pH} 4.50)$, but decreased $\mathrm{pH}$ in the absence of VFA did not significantly influence the conductivity. Therefore, changes in conductivity observed in the presence of various concentrations of VFA are most likely due to a different degree of dissociation of VFA and are not a direct effect of different proton concentrations. Increasing the osmolarity of the medium by addition of D-mannitol $(0,50,100,150$ and $200 \mathrm{mmol} / \mathrm{L})$ reduced conductivity $(0.55 \mathrm{mS}$ per $50 \mathrm{mmol}$ of added mannitol). This finding might be explained by a decreased mobility of ions at increasing concentrations of solutes.

All changes in conductivity recorded by the intraruminal device during the in vitro evaluation were highly correlated $\left(n=14\right.$; Pearson $\left.r=0.92, \mathrm{R}^{2}=0.84\right)$ with those values measured by an external conductivity meter (HI 9033 multi-range conductivity meter, HANNA instruments, Germany).

Taken together, recording of the conductivity appears to be principally useful to get information about the concentration of VFA, $\mathrm{pH}$ and osmolarity. However the in vivo situation may be much more complex due to overlapping effects of concentration and dissociation of VFA and osmolarity on conductivity.

Over the range of 1 to $120 \mathrm{hPa}$, the sensitivity of the integrated barometer was constant and pressure changes were recorded with an efficiency of $66 \%$. In vivo, pressure recordings were successfully used for registration of contractions of the reticulorumen during surgery (Figure 1).

For future use of the device as a management tool rumen contractions could be a valuable parameter to detect indigestion located in the forestomachs, adequacy of physical structure in the diet, and overfeeding with rapidly fermentable carbohydrates. 


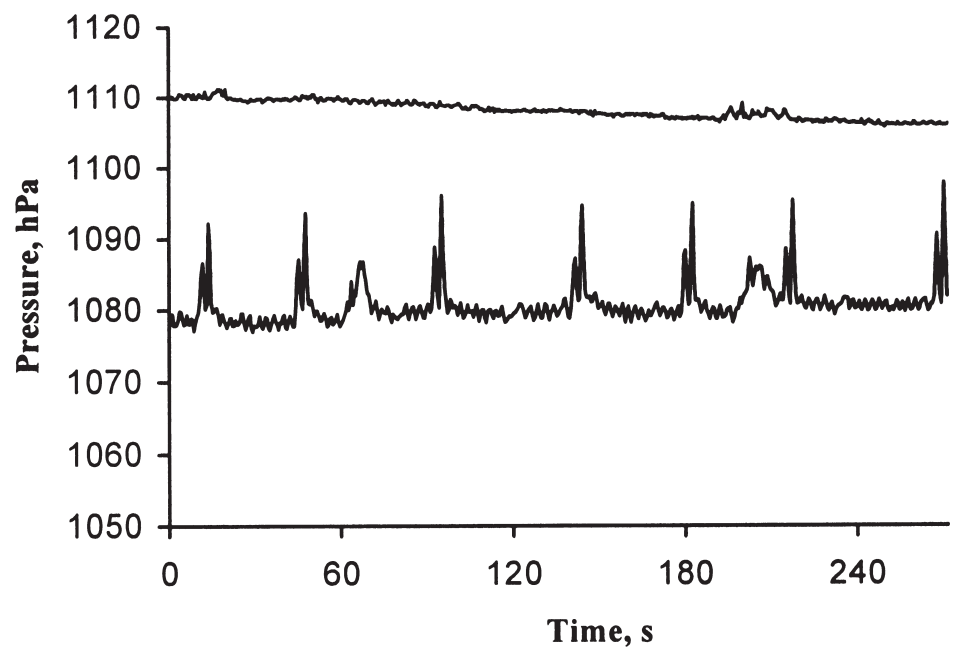

Figure 1. Recording of the intrareticular pressure changes by an intraruminal device during (upper trace) and after $5 \mathrm{~h}$ of recovery from (lower trace) anaesthesia (isoflurane)

\section{CONCLUSIONS}

The results obtained in the in vitro experiments indicate that changes in temperature, conductivity and pressure can be reliably recorded by the device. Furthermore these results as well as the results obtained from the in vivo experiment are promising for a further development of the device as a management tool for dairy cows.

\section{REFERENCES}

Lehrer A.R., Lewis G.S., Aizinbud E., 1992. Oestrus detection in cattle: recent developments. Anim. Reprod. Sci. 28, 355-361

Susenbeth A., Dickel T., Südekum, K.-H., Drochner W., Steingaß H., 2004. Energy requirements of cattle for standing and for ingestion, estimated by a ruminal emptying technique. J. Anim. Sci. 82, 129-136 